\section{Saúde sexual dos adolescentes segundo a Pesquisa Nacional de Saúde dos Escolares}

\section{Sexual health of adolescents according to the National Survey of School Health}

\section{Deborah Carvalho Malta ' \\ Marta Angélica lossi Silva " \\ Flávia Carvalho Malta de Mello "I \\ Rosane Aparecida Monteiro Iv \\ Denise Lopes Porto ${ }^{v}$ \\ Luciana Monteiro Vasconcelos Sardinha VI \\ Paula Carvalho de Freitas vil}

' Coordenação Geral de Doenças e Agravos Não-transmissíveis da Secretaria de Vigilância em Saúde do Ministério da Saúde - Brasília (DF); Universidade Federal de Minas Gerais (UFMG) - Belo Horizonte (MG), Brasil

"Escola de Enfermagem de Ribeirão Preto da Universidade de São Paulo (USP) Ribeirão Preto (SP), Brasil

II' Escola de Enfermagem de Ribeirão Preto da USP - Ribeirão Preto (SP), Brasil

Iv Faculdade de Medicina de Ribeirão Preto da USP - Ribeirão Preto (SP), Brasil

Departamento de Análise de Situação de Saúde da Secretaria de Vigilância em Saúde do Ministério da Saúde - Brasília (DF), Brasil

vı Coordenação Geral de Doenças e Agravos Não-transmissíveis da Secretaria de Vigilância em Saúde do Ministério da Saúde - Brasília (DF), Brasil

VII Coordenação Geral de Doenças e Agravos Não-transmissíveis da Secretaria de Vigilância em Saúde do Ministério da Saúde - Brasília (DF), Brasil

Trabalho realizado na Secretaria de Vigilância em Saúde do Ministério da Saúde. Fonte de financiamento: Ministério da Saúde.

Correspondência: Deborah Carvalho Malta - Coordenação Geral de Doenças e Agravos Nãotransmissíveis da Secretaria de Vigilância em Saúde do Ministério da Saúde - SAF Sul, Trecho 02, Lotes 05 e 06, Bloco F, Torre I, Edifício Premium - Térreo - Sala 14 - CEP: 70070-600 - Brasília (DF), Brasil - E-mail: deborah.malta@saude.gov.br.

Conflito de interesse: nada a declarar.

\section{Resumo}

O objetivo deste estudo foi descrever as situações relacionadas à saúde sexual dos adolescentes, segundo a Pesquisa Nacional de Saúde do Escolar (PeNSE). Trata-se de um estudo transversal, realizado pelo Instituto Brasileiro de Geografia e Estatística (IBGE) em parceria com o Ministério da Saúde, que envolveu 60.973 escolares e 1.453 escolas públicas e privadas. A análise dos dados aponta que $30,5 \%$ (IC95\% 29,9-31,2) dos adolescentes já tiveram relação sexual alguma vez na vida, sendo mais frequente em meninos (43,7\%; IC95\% 42,7-44,7) do que em meninas (18,7\%; IC95\% 18,0-19,4), naqueles que estudam em escola pública (33,1\%; IC95\% 32,4-33,9), com idade acima de 15 anos (47,3\%; IC95\% 45,7-48,9) e aos 16 anos (63,5\%; IC95\% 61,5-65,4). A idade de iniciação sexual foi precoce e $40,1 \%$ dos adolescentes (IC95\% 38,8-41,4) relataram ter tido um único parceiro na vida. $\mathrm{O}$ uso do preservativo na ultima relação sexual foi elevado tanto para proteção $(75,9 \%$; IC95\% 74,8-76,9), como também para método contraceptivo na última relação sexual (74,7\%; IC95\% 73,6-75,7). Torna-se necessário enfatizar ações de promoção à saúde sexual voltadas aos adolescentes, visando minimizar vulnerabilidades.

Palavras-chave: sexualidade; adolescente; instituições acadêmicas; epidemiologia descritiva. 


\section{Abstract}

The objective of this study was to describe situations related to sexual health of adolescents, according to the National Survey of School Health (PeNSE). It is a cross-sectional study performed by the Brazilian Institute of Geography and Statistics (IBGE), in partnership with the Ministry of Health that involved 60,973 students and 1,453 public and private schools. Data analysis points out that $30.5 \%$ (95\%CI 29.9-31.2) of the adolescents had already had sexual relations sometime in their lives, being more frequent for males (43.7\%; 95\%CI 42.7-44.7) than females (18.7\%; 95\%CI 18.0-19.4), especially those who go to public schools (33.1\%; 95\%CI 32.4-33.9), aged 15 years or older (47.3\%; 95\%CI 45.7-48.9) and 16 years (63.5\%; 95\%CI 61,5-65.4). The sexual initiation age was early and $40.1 \%$ (95\%CI 38.8-41.4) reported having had only one partner in life. The use of condoms in the last sexual relation was high both for protective (75.9\%; CI95\% 74.8-76.9) and contraceptive methods (74.7\%; 95\%CI 73.6-75.7). It is necessary to emphasize actions for promoting sexual health towards adolescents in order to minimize vulnerabilities.

Keywords: sexuality; adolescent; schools; epidemiology, descriptive.

\section{Introdução}

A adolescência constitui uma fase da vida de grandes transformações emocionais, cognitivas, sociais e corporais ${ }^{1}$. Destacam-se, ainda, as mudanças relativas ao relacionamento afetivo entre os jovens e a sexualidade ${ }^{1}$.

Entre os diversos problemas e questões que continuam a desafiar as políticas de desenvolvimento no Brasil e na América Latina, seguramente um dos mais importantes é a adolescência. Nas últimas décadas, esta vem sendo objeto de estudo de diferentes pesquisadores e sua importância, atualmente, está ligada a diversas dimensões e áreas como a educação, saúde, cultura, cidadania, violência e pobreza $^{2}$. A busca da identidade e do novo, a curiosidade, a onipotência e a contestação despertam no adolescente uma sensação de invulnerabilidade, a qual associada a pouca experiência de vida e a fatores socioculturais e econômicos, muitas vezes associados a fatores familiares, caracteriza este grupo como de potencial vulnerabilidade ${ }^{3-4}$.

Neste sentido e com as mudanças sociais vividas nas últimas décadas, acompanha-se o início da vida sexual dos adolescentes cada vez mais cedo. Sendo este fato determinado por muitas formas de desigualdade, se analisado por gênero, raça/cor, escolaridade e condição socioeconômica. Estas desigualdades influenciam as relações de saúde desta população, acarretando em piora dos indicadores de morbimortalidade ${ }^{5}$. A iniciação sexual precoce está associada com o não-uso ou uso inadequado dos preservativos e suas consequências, ou seja, gravidez precoce, doenças sexualmente transmissíveis (DST), AIDS; além de estar associado com o uso do tabaco, consumo de álcool e outras drogas ${ }^{1}$.

A gravidez não-intencional é uma das grandes consequências negativas da iniciação sexual entre adolescentes. No caso da gravidez levada a termo, consequências adversas podem ser esperadas 
tanto para a criança quanto para a $\mathrm{mãe} \mathrm{e}^{1}$.

Visando monitorar essas ações junto a este público, em 2009, o Ministério da Saúde e o Instituto Brasileiro de Geografia e Estatística (IBGE) constituíram parceria para a realização da primeira Pesquisa Nacional de Saúde do Escolar (PeNSE) ${ }^{6}$. O objetivo da pesquisa foi ampliar o conhecimento sobre a saúde desta população, gerando evidências para orientar ações de promoção da saúde neste grupo etário. A pesquisa contou ainda com o apoio institucional do Ministério da Educação.

O monitoramento sobre a saúde dos adolescentes já está implantado em mais de 40 países no mundo e tem auxiliado na modificação de currículos e reestruturação dos programas de saúde voltados para essa faixa etária ${ }^{7-9}$.

A PeNSE foi a primeira iniciativa nacional realizada, perguntando diretamente aos adolescentes sobre os fatores de risco e proteção, constituindo-se importante instrumento de apoio aos gestores, pais, profissionais de saúde e educadores $^{10}$.

O objetivo deste trabalho é descrever as situações relacionadas à saúde sexual dos adolescentes, segundo a PeNSE.

\section{Métodos}

Trata-se de um estudo transversal descritivo, realizado com escolares do nono ano (oitava série) do Ensino Fundamental, de escolas públicas e privadas das capitais dos estados brasileiros e do Distrito Federal, entre março a junho de 2009.

Foi desenhada uma amostra de alunos por conglomerados em dois estágios; no primeiro estágio, foi feita a seleção das escolas e, no segundo, a seleção das turmas, entrevistando os alunos das turmas selecionadas ${ }^{10}$.

A amostra foi calculada para fornecer estimativas de proporções (ou prevalências) de algumas características de interesse, em cada um dos estratos geográficos, com um erro máximo de $3 \%$ em valor absoluto ao nível de confiança de $95 \%^{10}$.

Foram amostradas 1.453 escolas e 2.175 turmas, nas quais haviam 68.735 alunos frequentes, sendo que 63.411 estavam presentes no dia da coleta, totalizando 7,7\% de perdas nesta etapa. Foram excluídos da amostra 501 estudantes que se negaram a participar e os que não preencheram a variável sexo. Assim, foram analisados dados referentes a 60.973 escolares, com uma taxa de não-resposta geral de $11,3 \%^{10,11}$.

Em cada uma das turmas do nono ano (oitava série) selecionadas, todos os alunos foram convidados a responder ao questionário da pesquisa, os que não se sentiam motivados a preencher o questionário eram dados como perda.

Foi utilizado o Personal Digital Assistant (PDA) para coleta dos dados individuais. O questionário estruturado autoaplicável contém módulos temáticos sobre diversos fatores de risco e proteção, dentre eles, o tema da sexualidade e orientações recebidas na escola sobre DST, AIDS e uso de preservativo.

Foram analisadas situações relacionadas à saúde sexual de adolescentes escolares do nono ano. A variável 'ter tido relação sexual alguma vez na vida' foi descrita segundo sexo, idade, escolaridade materna, raça/cor e estudar em escola pública e privada. Esta variável foi ainda descrita pelas capitais do país, segundo sexo.

Em relação àqueles que descreveram já ter tido relação sexual, foram pesquisadas e analisadas as seguintes variáveis: idade da primeira relação sexual; número de parceiros na vida, etc.; ter tido relação sexual no último ano; uso de preservativo na última relação e uso de método para evitar a gravidez na última relação. Em todas as variáveis foram descritas as frequências (proporções) e seus respectivos intervalos de confiança (IC95\%).

O estudo foi aprovado no Conselho de Ética em Pesquisas do Ministério da Saúde, 
sob a emenda no $005 / 2009$, referente ao Registro no 11.537 da Comissão Nacional de Ética em Pesquisa do Ministério da Saúde (CONEP/MS), em 10 de junho de 2009. O Estatuto da Criança e do Adolescente prevê autonomia do adolescente para tomar iniciativas, tais como responder a um questionário que não ofereça riscos a sua saúde. Como a pesquisa visa subsidiar políticas de proteção à saúde para esta faixa etária, optou-se pela autonomia do adolescente em definir sobre sua participação na pesquisa. O estudante escolhia em participar, ou não, e poderia responder o questionário ou apenas parte do mesmo. O termo de consentimento do aluno foi dado no próprio PDA pelo próprio aluno. As informações do aluno foram confidenciais e não-identificadas, bem como as da escola $^{11}$.

A realização do estudo foi precedida do contato com as Secretarias Estadual e Municipal de Educação e com a direção das escolas selecionadas em cada município.

\section{Resultados}

Os resultados revelaram que $30,5 \%$ (IC95\%29,9-31,2)dosadolescentesjátiveram relação sexual alguma vez na vida, sendo mais meninos (43,7\%; IC95\% 42,7-44,7) do que meninas, (18,7\%; IC95\% 18,0-19,4), com maiores percentuais nas escolas públicas (33,1\%; IC95\% 32,4-33,9) do que nas privadas (20,7\%; IC95\% 19,7-21,8). A frequência por idade mostra que, antes dos 13 anos, 12,5\% (IC95\% 9,4-16,5) já haviam tido relação sexual; aos 13 anos, 15,3\% (IC95\% 14,3-16,5); aos 14 anos, $24,9 \%$ (IC95\% 24,0-25,8); aos 15 anos, 47,3\% (IC95\% 45,7-48,9) e aos 16 anos, 63,5\% (IC95\% 61,5-65,4). Os adolescentes de cor branca relatam menor frequência $(26,2 \%$; IC95\% 25,2-27,2), quando comparados com os de cor negra (39,8\%; IC95\% 37,9-41,7) e parda (31,6\%; IC95\% 30,6-32,6). Quanto maior a escolaridade materna, menor o percentual de escolares que já tiveram relação sexual alguma vez na vida.
Observou-se que 42,1\% (IC95\% 39,4-44,9) dos adolescentes que não residem com o pai e nem com mãe já tiveram relação sexual alguma vez na vida, reduzindo para 26,6\% (IC95\% 25,8-27,4) quando residem com pai e mãe (Tabela 1 ).

Dos escolares do nono ano do Ensino Fundamental que já tiveram relação sexual alguma vez na vida, por sexo e capitais de estados e Distrito Federal, a PeNSE revelou que entre as capitais que apresentam frequências acima da média nacional para o sexo feminino estiveram: Salvador (25,7\%; IC95\% 23,0-28,3), Boa Vista (25,7\%; IC95\% 22,8-28,6), Manaus (24,0\%; IC95\% 21,0-27,0), Belém (24,0\%; IC95\% 21,2-26,8), Macapá (22,6\%; IC95\% 20,3-24,9), Porto Velho (22,3\%; IC95\% 19,8-24,8), Florianópolis (22,2\%; IC95\% 19,7-24,8), Campo Grande (22,1\%; IC95\% 19,5-24,7). João Pessoa (13,9\%; IC95\% 11,7-16,1) e Teresina (14,3\%; IC95\% 12,3-16,3) foram as capitais em que as meninas apresentaram o menor percentual (Figura 1).

O estudo mostrou, entre os estudantes do sexo masculino do nono ano que já tiveram relação sexual alguma vez na vida, as capitais que apresentaram frequências abaixo da média nacional: São Paulo (39,1\%; IC95\% 36,3-41,9), Fortaleza (38,6\%; IC95\% 35,35-41,8), Distrito Federal (37,7\%; IC95\% 34,9-40,5), Florianópolis (34,8\%; IC95\% 31,8-37,7) e Vitória (34,7\%; IC95\% 31,7-37,7). E as capitais com médias acima da nacional foram: Boa Vista (56,6\%; IC95\% 53,2-60,1), Macapá (55,0\%; IC95\% 51,9-58,1), Salvador (53,7\%; IC95\% 50,2-57,1), São Luiz (52,0\%; IC95\% 49,0-55,0), Manaus (51,4\%; IC95\% 47,6-55,2), Belém (51,0\%; IC95\% 47,6-54,4), Rio Branco (50,9\%; IC95\% 47,3-54,5), Recife (50,8\%; IC95\% 47,7-53,9), Rio de Janeiro (49,5\%; IC95\% 46,7-52,4), Cuiabá (49,5\%; IC95\% 46,0-53,0), Porto Velho (48,9\%; IC95\% 45,4-52,4) e Maceió (48,8\%; IC95\% 44,8-52,8).

Os resultados nas capitais brasileiras revelam que a idade da primeira 
Tabela 1. Percentual (\% e IC95\%)* de escolares do nono ano do Ensino Fundamental que já tiveram relação sexual alguma vez na vida, segundo idade, sexo, raça/cor, escolaridade materna e com quem reside da população de escolares do conjunto das capitais dos estados brasileiros e Distrito Federal

Table 1. Percentage (\% and $95 \% \mathrm{Cl}) *$ of students at freshman year high school that already had sexual relations sometime in their life, according to age, gender, ethnicity/color, schooling of the mother, with whom resides, in the student population from the set of Brazilian state capitals and the Federal District

\begin{tabular}{|c|c|c|c|c|c|c|c|c|c|}
\hline \multirow{2}{*}{ Relação sexual alguma vez na vida } & \multicolumn{2}{|c|}{ Total } & \multicolumn{3}{|c|}{ Escola Pública } & \multicolumn{4}{|c|}{ Escola Privada } \\
\hline & $\%$ & IC95\% & & $\%$ & $\mathrm{IC} 95 \%$ & & $\%$ & IC95\% & \\
\hline \multicolumn{10}{|l|}{ Idade } \\
\hline$<13$ & 12,5 & 9,4 & 16,5 & 12,7 & 9,0 & 17,3 & 12,2 & 7,3 & 19,9 \\
\hline 13 & 15,3 & 14,3 & 16,5 & 16,7 & 15,4 & 18,2 & 12,0 & 10,5 & 13,6 \\
\hline 14 & 24,9 & 24,0 & 25,8 & 26,4 & 25,4 & 27,5 & 19,8 & 18,4 & 21,3 \\
\hline 15 & 47,3 & 45,7 & 48,9 & 47,5 & 45,8 & 49,2 & 45,4 & 41,2 & 49,6 \\
\hline 16 e mais & 63,5 & 61,5 & 65,4 & 63,6 & 61,6 & 65,6 & 61,7 & 55,1 & 67,9 \\
\hline \multicolumn{10}{|l|}{ Sexo } \\
\hline Masculino & 43,7 & 42,7 & 44,7 & 46,8 & 45,6 & 48,0 & 32,4 & 30,7 & 34,1 \\
\hline Feminino & 18,7 & 18,0 & 19,4 & 21,0 & 20,1 & 21,9 & 9,8 & 8,7 & 11,0 \\
\hline \multicolumn{10}{|l|}{ Cor ou raça } \\
\hline Branca & 26,2 & 25,2 & 27,2 & 29,7 & 28,4 & 31,0 & 18,4 & 17,1 & 19,8 \\
\hline Parda & 31,6 & 30,6 & 32,6 & 32,8 & 31,7 & 33,9 & 24,0 & 22,0 & 26,2 \\
\hline Preta & 39,8 & 37,9 & 41,7 & 40,9 & 38,9 & 43,0 & 29,4 & 25,2 & 34,0 \\
\hline Amarela & 29,2 & 26,1 & 32,4 & 34,2 & 30,4 & 38,3 & 17,2 & 13,0 & 22,4 \\
\hline Indígena & 35,5 & 32,6 & 38,5 & 37,7 & 34,3 & 41,1 & 26,7 & 21,0 & 33,2 \\
\hline \multicolumn{10}{|l|}{ Escolaridade materna } \\
\hline $\begin{array}{l}\text { Nenhuma ou ensino fundamental } \\
\text { incompleto }\end{array}$ & 35,2 & 33,9 & 36,5 & 35,4 & 34,0 & 36,7 & 31,9 & 26,6 & 37,6 \\
\hline $\begin{array}{l}\text { Ensino fundamental completo ou } \\
\text { médio incompleto }\end{array}$ & 32,9 & 31,1 & 34,6 & 34,0 & 32,1 & 35,9 & 24,2 & 20,3 & 28,5 \\
\hline $\begin{array}{l}\text { Ensino médio completo ou } \\
\text { superior incompleto }\end{array}$ & 29,2 & 28,0 & 30,5 & 31,7 & 30,2 & 33,2 & 21,4 & 19,5 & 23,4 \\
\hline Ensino superior completo & 22,9 & 21,5 & 24,3 & 30,9 & 28,3 & 33,7 & 17,7 & 16,3 & 19,1 \\
\hline \multicolumn{10}{|l|}{ Com quem reside } \\
\hline Residir com pai e mãe & 26,6 & 25,8 & 27,4 & 29,2 & 28,3 & 30,2 & 18,1 & 16,9 & 19,4 \\
\hline Só mãe & 34,6 & 33,4 & 35,7 & 36,7 & 35,4 & 38,1 & 24,3 & 22,1 & 26,6 \\
\hline Só pai & 38,2 & 35,2 & 41,3 & 39,8 & 36,4 & 43,3 & 31,1 & 25,0 & 37,9 \\
\hline Nem pai e mãe & 42,1 & 39,4 & 44,9 & 44,0 & 40,9 & 47,1 & 31,3 & 26,2 & 36,9 \\
\hline Total & 30,5 & 29,9 & 31,2 & 33,1 & 32,4 & 33,9 & 20,7 & 19,7 & 21,8 \\
\hline
\end{tabular}

* Percentual ponderado para representar a população de escolares matriculados e frequentando o nono ano do Ensino Fundamental em 2009. IC95\%: intervalo de confiança de $95 \%$

*Weighted percentage to represent the population of students going to the freshman year in $2009.95 \% \mathrm{Cl}$ : $95 \%$ confidence interval.

relação sexual foi predominantemente nas idades de 13 (26,1\%; IC95\% 24,9-27,3) e 14 anos (26,5\%; IC95\% 25,4-27,7). Chama a atenção o fato de os escolares terem relatado a primeira relação sexual aos 9 (6,9\%; IC95\%=6,47,6), 10 $(4,6 \%$; IC95\% 4,1-5,2) e 11 anos $(6,3 \%$; IC95\% 5,6-7,0), sempre maior entre meninos. A maioria dos escolares relatou apenas um $(40,1 \%$; IC95\% 38,8-41,4) ou dois parceiros (18,8\%; IC95\% 17,8-19,9). A grande maioria teve relação no último ano (69\%; IC95\% 67,8-70,1). A frequência do uso de preservativo na última relação sexual foi de 75,9\% (IC95\% 74,8-76,9), sendo maior entre meninos $(77 \%$; IC95\% 75,7-78,3) do que entre meninas $(73,5 \%$; IC95\% 71,6-75,4). Na última 
1A. Feminio

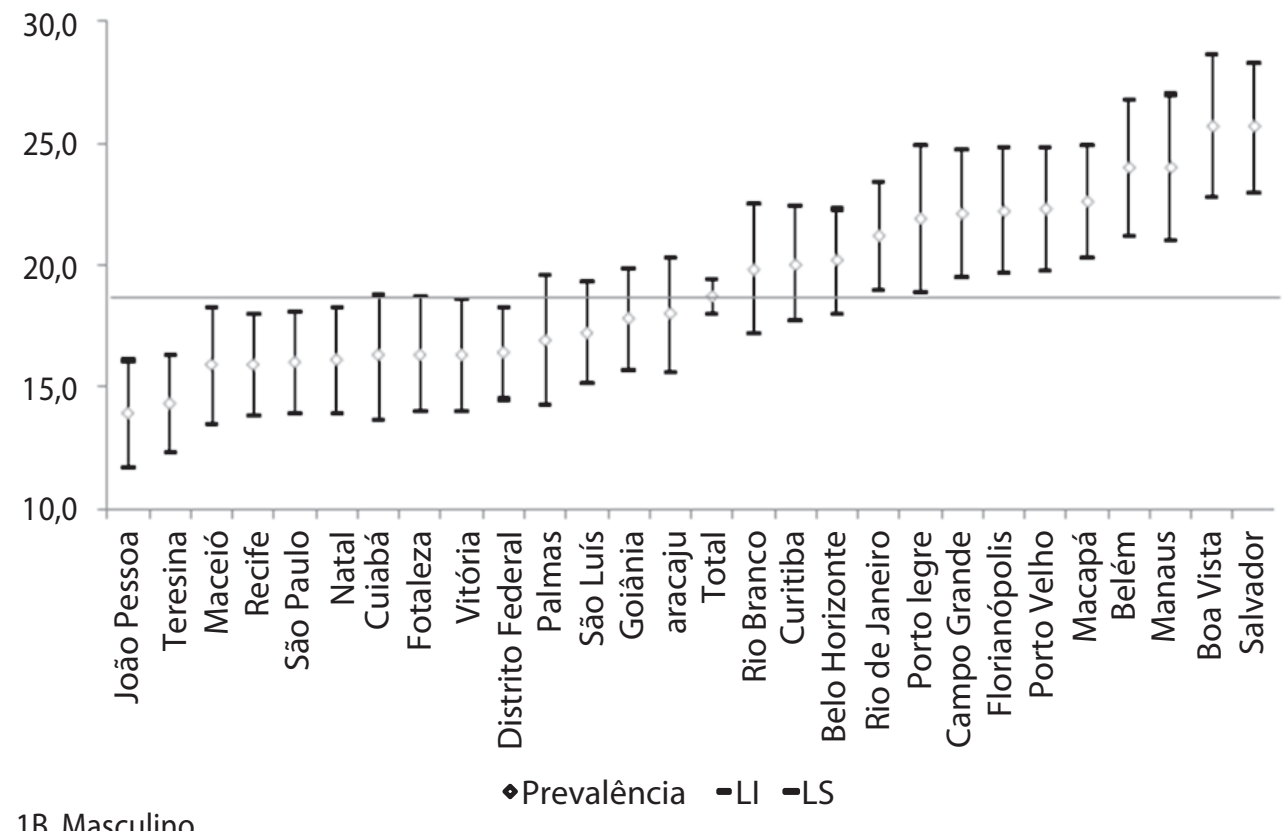

1B. Masculino

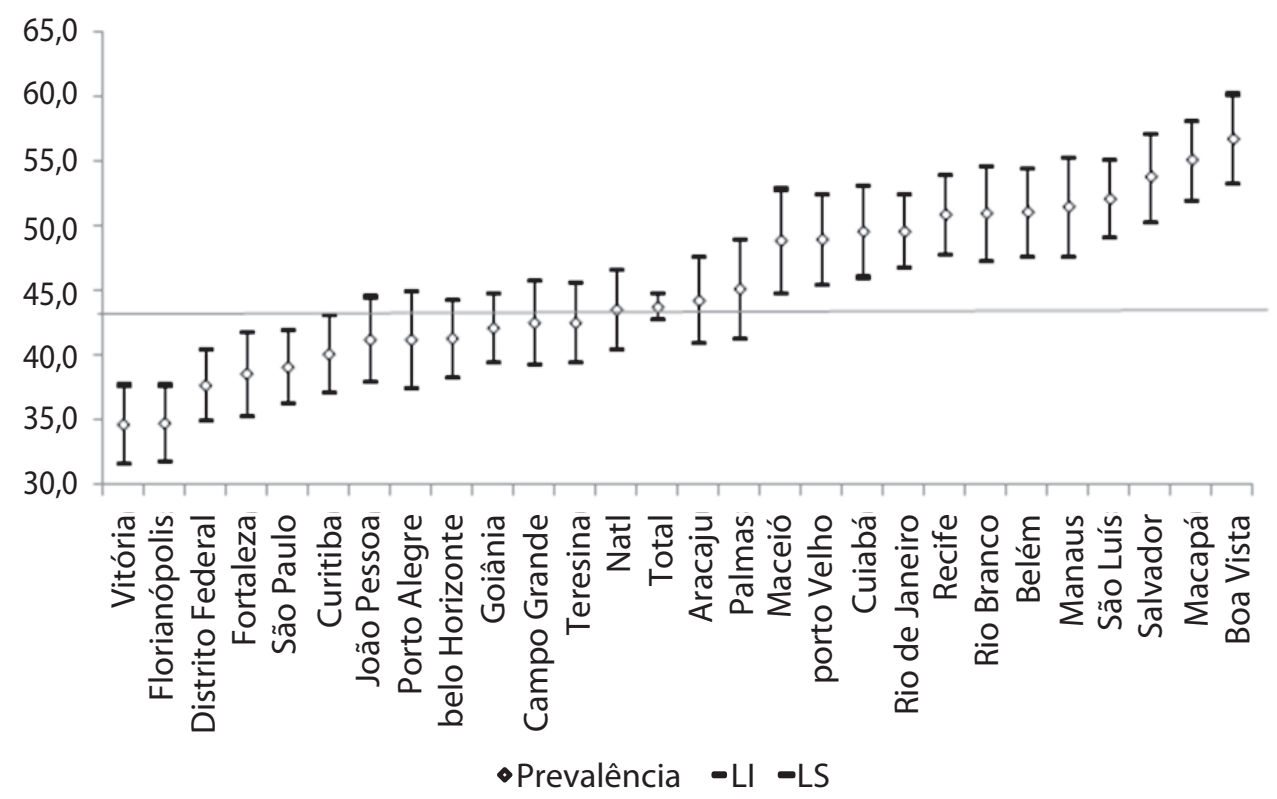

* Percentual ponderado para representar a população de escolares matriculados e frequentando o nono ano do Ensino Fundamental, em 2008. IC95\%: intervalo de confiança de 95\%

*Weighted percentage to represent the population of students going to the freshman year of high school in $2008.95 \% \mathrm{Cl}: 95 \%$ confidence interval.

Figura 1. Percentual (\% e IC95\%)* de escolares do nono ano do Ensino Fundamental que já tiveram relação sexual alguma vez na vida, por sexo e capitais de estados e Distrito Federal Figure 1. Percentage of students at freshman year high school that already had sexual relations sometime in their life gender, and capitals and the Federal District 
Tabela 2. Frequência* (e respectivos IC95\%) de situações selecionadas à saúde sexual, referidas pelos escolares do nono ano do Ensino Fundamental, que já tiveram relação sexual alguma vez na vida, segundo sexo

Table 2. Frequency* (and respective $95 \% \mathrm{Cl}$ ) of selected sexual health situations referred by students at freshman year high school that already had sexual relations sometime in their life, according to gender

\begin{tabular}{|c|c|c|c|c|c|c|c|c|c|}
\hline \multirow{3}{*}{$\begin{array}{l}\text { Relação sexual alguma vez na vida } \\
\text { Idade da primeira relação sexual }\end{array}$} & \multicolumn{3}{|c|}{ Total } & \multicolumn{3}{|c|}{ Masculino } & \multicolumn{3}{|c|}{ Feminino } \\
\hline & \multirow[t]{2}{*}{$\%$} & \multicolumn{2}{|c|}{ IC 95\% } & \multirow[t]{2}{*}{$\%$} & \multicolumn{2}{|c|}{ IC $95 \%$} & \multirow[t]{2}{*}{$\%$} & \multicolumn{2}{|c|}{ IC 95\% } \\
\hline & & & & & & & & & \\
\hline 9 anos ou menos & 6,9 & 6,4 & 7,6 & 9,2 & 8,4 & 10,1 & 2,0 & 1,5 & 2,6 \\
\hline 10 anos & 4,6 & 4,1 & 5,2 & 6,2 & 5,5 & 7,0 & 1,3 & 0,9 & 2,0 \\
\hline 11 anos & 6,3 & 5,6 & 7,0 & 7,7 & 6,8 & 8,6 & 3,3 & 2,5 & 4,3 \\
\hline 12 anos & 13,1 & 12,2 & 14,0 & 15,0 & 13,9 & 16,1 & 8,9 & 7,7 & 10,2 \\
\hline 13 anos & 26,1 & 24,9 & 27,3 & 25,4 & 24,0 & 26,9 & 27,6 & 25,6 & 29,7 \\
\hline 14 anos & 26,5 & 25,4 & 27,7 & 24,0 & 22,6 & 25,4 & 32,1 & 30,1 & 34,2 \\
\hline 15 anos & 11,9 & 11,2 & 12,8 & 9,2 & 8,4 & 10,1 & 17,9 & 16,2 & 19,6 \\
\hline 16 anos ou mais & 4,5 & 4,0 & 4,9 & 3,3 & 2,9 & 3,8 & 7,0 & 6,0 & 8,0 \\
\hline \multicolumn{10}{|l|}{ Número de parceiros na vida } \\
\hline 1 pessoa na vida & 40,1 & 38,8 & 41,4 & 31,3 & 29,9 & 32,8 & 59,0 & 56,8 & 61,2 \\
\hline 2 pessoas na vida & 18,8 & 17,8 & 19,9 & 20,2 & 18,9 & 21,5 & 16,0 & 14,4 & 17,7 \\
\hline 3 pessoas na vida & 11,5 & 10,7 & 12,4 & 12,9 & 11,9 & 14,1 & 8,4 & 7,3 & 9,7 \\
\hline 4 pessoas na vida & 6,0 & 5,4 & 67,0 & 6,9 & 6,2 & 7,8 & 4,2 & 3,3 & 5,2 \\
\hline 5 pessoas na vida & 3,7 & 3,2 & 4,3 & 4,1 & 3,5 & 4,8 & 2,9 & 2,2 & 3,9 \\
\hline 6 ou mais pessoas na vida & 10,3 & 9,5 & 11,1 & 12,9 & 11,8 & 14,0 & 4,6 & 3,8 & 5,6 \\
\hline Não me lembro & 9,5 & 8,8 & 10,3 & 11,7 & 10,7 & 12,7 & 4,8 & 4,0 & 5,8 \\
\hline \multicolumn{10}{|l|}{ Relação sexual no último ano } \\
\hline Sim & 69,0 & 67,8 & 70,1 & 65,1 & 63,6 & 66,5 & 77,2 & 75,4 & 78,9 \\
\hline Não & 31,0 & 29,9 & 32,2 & 34,9 & 33,5 & 36,4 & 22,8 & 21,1 & 24,6 \\
\hline \multicolumn{10}{|l|}{$\begin{array}{l}\text { Uso de preservativo na última } \\
\text { relação }\end{array}$} \\
\hline Sim & 75,9 & 74,8 & 76,9 & 77,0 & 75,7 & 78,3 & 73,5 & 71,6 & 75,4 \\
\hline Não & 21,3 & 20,3 & 22,3 & 19,8 & 18,6 & 21,1 & 24,4 & 22,6 & 26,3 \\
\hline \multicolumn{10}{|l|}{$\begin{array}{l}\text { Uso de método para evitar a } \\
\text { gravidez na última relação }\end{array}$} \\
\hline Sim & 74,7 & 73,6 & 75,7 & 73,3 & 71,9 & 74,7 & 77,5 & 75,6 & 79,2 \\
\hline Não & 19,5 & 18,5 & 20,4 & 19,3 & 18,1 & 20,5 & 19,9 & 18,2 & 21,6 \\
\hline
\end{tabular}

relação, também foi elevado o uso de método para evitar gravidez $(74,7 \%$; IC95\% 73,6-75,7), e foi maior entre o sexo feminino (77,5\%; IC95\% 75,6-79,2) do que o masculino (73,3\%; IC95\% 71,9-74,7), como pode ser observado na Tabela 2.

\section{Discussão}

De acordo com os dados da PeNSE, aproximadamente um terço dos adolescentes já haviam tido relação sexual alguma vez na vida, sendo mais meninos do que meninas e mais aqueles que estudam em escola pública. A idade da relação sexual foi precoce, e a maioria relatou ter tido um único parceiro na vida. O uso do preservativo e de método contraceptivo foi elevado.

Comparações internacionais mostram que a idade da iniciação sexual varia entre países. Uma pesquisa realizada em 2005 a 2006, pela Organização Mundial de Saúde (OMS) ${ }^{1}$, revelou uma média de $22 \%$ de adolescentes que já haviam realizado relação sexual aos 15 anos, sendo esta proporção de $5 \%$, na Macedônia, a $61 \%$, na Groelândia, mostrando diferenças culturais importantes.

Nos Estados Unidos, um inquérito realizado em 2007 entre alunos do $9^{\circ}$ até o $12^{\circ}$ ano (equivalente ao Ensino Médio) verificou que $47,8 \%$ dos escolares já 
tiveram iniciação sexual e 89,5\% dos alunos receberam, na escola, orientação sobre DST/AIDS ${ }^{12}$.

Os dados da pesquisa atual devem ser contextualizados em relação às pesquisas internacionais, em função da faixa etária. $\mathrm{Na}$ PeNSE, a amostra é constituída por alunos entre 13 a 15 anos (88\%), nas pesquisas da OMS, os alunos que respondem a este bloco são maiores de 15 anos e nos Estados Unidos são alunos da High School, entre 14 a 17 anos, quando espera-se maior proporção de iniciação sexual.

Em pesquisa nacional representativa da população urbana brasileira sobre iniciação sexual verificou-se que $61 \%$ dos jovens avaliados em 1998 e 61,6\%, em 2005 já haviam tido relacionamento sexual ${ }^{13}$. Por outro lado, um estudo realizado na cidade de São Paulo entre adolescentes de 15 a 19 anos revelou que $46,1 \%$ já tinham iniciação sexual ${ }^{14}$.

Na capital Rio Branco, no Acre, em inquérito escolar com estudantes do Ensino Médio, 48,9\% deles relataram já ter iniciação sexual, sendo a maioria (52,3\%), alunos oriundos de escolas particulares ${ }^{15}$. Este fato difere dos resultados da PeNSE, quando os alunos de escola pública tiveram maior frequência da relação sexual $(33,1 \%)$ do que os de escola privada $(20,8 \%)$. Esta diferença, na maior iniciação sexual nas escolas públicas, pode ser explicada pela diferença etária: nas privadas, 95,9\% dos alunos têm entre 13 a 15 anos e, na pública, $87,3 \%$. Os alunos de 13 anos somam $33,4 \%$ dos estudantes da escola privada e 21,2\%, na rede pública. Enquanto os alunos de 16 anos e mais somam 3,1\% nas escolas privadas, representam apenas $12,1 \%$ nas escolas públicas. Estes dados não-mostrados podem ajudar a explicar a maior frequência de iniciação sexual entre alunos de escola pública, sendo que alunos mais velhos têm maior chance da iniciação sexual na vida.

Discutir acerca da iniciação sexual na adolescência é também considerar uma discussão acerca da sexualidade na adolescência a qual deve ser compreendida a partir de uma perspectiva sócio-histórica ${ }^{16}$.
É importante considerar, não somente o caráter natural da sexualidade, mas entendê-la como uma construção cultural. Enquanto dado cultural, pode-se perceber que o exercício da sexualidade implica em conhecimentos, aprendizados, habilidades e possibilidades sociais concretas, advindas de concepções, valores, atitudes e habilidades sociais, as quais variam de sujeito para sujeito e de grupo para grupo ${ }^{17}$.

Dentre estas possibilidades, pode-se destacar os estímulos à iniciação sexual veiculados pela mídia e à pressão grupal, considerando-se, sobretudo, as questões referidas ao gênero masculino, no qual esta iniciação precoce é altamente estimulada, há maior tolerância à maternidade solteira $\mathrm{e}$ uma diferente valorização da virgindade na adolescência $^{18-19}$.

$\mathrm{Na}$ presente pesquisa, dos alunos que já haviam tido relação sexual, cerca de $76 \%$ usaram preservativo na última relação sexual. A incidência e a prevalência das DSTs têm aumentado, mesmo entre adolescentes $^{20}$. Estes são vulneráveis às infecções pelo HIV, sendo que $25 \%$ dos novos infectados no mundo são menores de 21 anos $^{1,21}$.

$\mathrm{O}$ uso de preservativos protege contra uma gravidez indesejada e também das DSTs, incluindo HIV. Preservativos são os métodos de contracepção mais usados entre os jovens. $\mathrm{O}$ não-uso dos preservativos constitui um marcador da relação sexual de risco. Outras condutas consideradas de risco são: iniciação sexual precoce, múltiplos parceiros e uso de álcool e drogas antes do sexo ${ }^{1}$.

A OMS divulgou inquérito em mais de 40 países em 2005 e 2006, entre adolescentes com 15 anos, no qual $77 \%$ dos adolescentes relatam ter usado preservativo na última relação, sendo maior entre homens $(81 \%)$ do que entre mulheres $(72 \%)^{22}$. Na Espanha, em inquérito para população escolar de 15 anos, $78,8 \%$ de homens e $82,0 \%$ de mulheres disseram ter usado preservativo na última relação sexual ${ }^{23}$. Nos Estados Unidos, um inquérito realizado em 2007, entre alunos do $9^{\circ}$ até o $12^{\circ}$ ano (equivalente ao Ensino Médio), verificou que entre escolares que já 
tinham tido relação sexual, $61,5 \%$ usaram preservativo durante a última relação sexual $^{12}$.

Portanto, os dados aqui apresentados mostram que, no Brasil, o uso de preservativo entre jovens está próximo aos estudos internacionais, situando-se inclusive acima do comportamento dos jovens norte-americanos, mostrando que as campanhas educativas e a mensagem têm chegado entre o público-alvo. Entretanto, ainda cerca de $25 \%$ não o utilizaram na última relação, embora mais de $80 \%$ tenham relatado que receberam orientação sobre prevenção de AIDS/DST e gravidez.

A importância da atenção e da educação preventiva relativa à saúde sexual e da orientação sexual para adolescentes tem sido oficialmente reconhecida por diversas organizações e instituições nacionais e internacionais. No Brasil, o Ministério da Saúde por intermédio do departamento de DST/AIDS e hepatites virais e o Ministério da Educação, implantaram, desde 2003, o Projeto Saúde e Prevenção nas Escolas (SPE), que consiste em integrar os setores da educação e saúde para o desenvolvimento de ações voltadas à promoção das saúdes sexual ereprodutiva de adolescentesejovens e para a redução de sua vulnerabilidade às
DSTs e à AIDS. Em 2008, com a criação pelo Governo Federal, do Programa Saúde na Escola (PSE), estas ações foram estendidas para um significativo conjunto de escolas e espera-se uma ampliação da abordagem da saúde sexual e reprodutiva no contexto das escolas do País.

\section{Conclusão}

Com base nos resultados apresentados, pode-se constatar que os adolescentes brasileiros têm iniciado suas relações sexuais cada vez mais cedo, o que leva à necessidade de promover a esta população a orientação, o apoio e a proteção adequados para o início desta nova experiência, a fim de que saiba-se lidar com a situação com mais responsabilidade, segurança e tranquilidade. Neste sentido, o apoio da família, da escola e dos profissionais da saúde é fundamental. Políticas públicas, programas e projetos que enfatizam e abordam a educação sexual, enquanto orientação para a vida, e tenham como eixo norteador a vulnerabilidade, a qual possibilita entender e interagir os diferentes aspectos individuais, programáticos e sociais, devem ser implantados para preparar os sujeitos para viverem no mundo presente e futuro.

\section{Referências}

1. World Health Organization. Inequalites in young people's health. Health Behavior in School- Aged Children.

International Report from 2005-2006. Health Police for Children and Adolescents, n. 5; 2008.

2. Waiselfisz JJ, Xavier R, Maciel M, Barbosa PD. Relatório de desenvolvimento juvenil - 2003. Brasília: UNESCO; 2004.

3. Alves AS, Lopes MHBM. Conhecimento, atitude e prática do uso de pílula e preservativo entre adolescentes universitários. Rev Bras Enferm. 2008;61(1):11-7.

4. Costa MCO, Bigras M. Mecanismos pessoais e coletivos de proteção e promoção da qualidade de vida para a infância e adolescência. Ciênc Saúde Colet. 2007;12(5):1101-9.

5. Brasil. Ministério da Saúde. Secretaria de Atenção à Saúde. Departamento de Ações Programáticas Estratégicas. Marco teórico e referencial: saúde sexual e saúde reprodutiva de adolescentes e jovens / Ministério da Saúde, Secretaria de Atenção à Saúde, Departamento de Ações Programáticas
Estratégicas. - Distrito Federal: Editora do Ministério da Saúde; 2006.

6. Brasil. Pesquisa Nacional de Saúde do Escolar. PENSE 2009. Rio de Janeiro: IBGE; [site na Internet]. 2009 [acessado 2010 abr 25]; Disponível em: http://www.ibge.gov.br/home/ estatistica/populacao/pense/default.shtm.

7. World Health Organization/United Nations Children's Fund/FOCUS. Measurement of adolescent development: environmental, contextual and protective factors. Geneva: World Health Organization; 1999.

8. Brener ND, Kann L, Kinchen SA, Grunbaum JA, Whalen L, Eaton D, et al. Methodology of the youth risk behavior surveillance system. MMWR Recomm Rep. 2004;53:1-13.

9. Currie C, Roberts C, Morgan A, Smith R, Settertobulte W, Samdal O, et al., editors. Young people's health in context. Health Behaviour in Schoolaged Children (HBSC) study: international report from the 2001/2002 survey. Geneva: World Health Organization; 2004. 
10. IBGE. Pesquisa Nacional de Saúde do Escolar 2009. Rio de Janeiro: IBGE; 2009. 144 p. [cited 2010 agosto]. Disponível em: <http://www.ibge.gov.br/home/ estatistica/populacao/ pense/pense.pdf $>$.

11. Malta DC, Sardinha LMV, Mendes I, Barreto SM, Giatti L, Castro IRR, et al. Prevalência de fatores de risco e proteção de doenças crônicas não transmissíveis em adolescentes: resultados da Pesquisa Nacional de Saúde do Escolar (PeNSE), Brasil, 2009. Ciênc Saúde Colet. 2010;15 Suppl 2:3009-19.

12. YRBS. Youth Risk Behavior Survey. Trends in the prevalence of sexual behavior. National YRBS: 1991-2007. Atlanta, GA: Centers for Disease Control and Prevention; 2007. [cited 2009 nov]. Disponível em: http://www.cdc.gov/HealthyYouth/ yrbs/pdf/yrbs07_us_sexual_behaviors_trend.pdf.

13. Brasil. 2000. Levantamento nacional sobre DST/AIDS nas escolas. [cited 2009 out]. http://bvsms.saude.gov.br/bvs/ publicacoes/33levantamento_nacional.pdf.

14. Borges ALV, Scha N. Início da vida sexual na adolescência e relações de gênero: um estudo transversal em São Paulo, Brasil 2002. Cad Saúde Pública. 2005;21(2):499-507.

15. Nascimento LCS, Lopes CM. Atividade sexual e doenças sexualmente transmissíveis em escolares do $2^{\circ}$ grau de Rio Branco-Acre, Brasil. Rev Lat Am Enf. 2000;8(1):107-13.

16. Bozon M. Sociologia da sexualidade. Rio de Janeiro: FGV; 2004.
17. Butler J. Cuerpos que importan: sobre os limites materiales y discusivos del "sexo". Buenos Aires: Paidós; 2005.

18. Gubert D, Madureira VSF. Iniciação sexual de homens adolescentes. Ciênc Saúde Col. [periódico na Internet]. 2009; [citado 2010 Dez 21];14(4). Disponível em: http:// www.scielo.br/scielo.php?script=sci_arttext\&pid=S1413$81232009000400018 \& \operatorname{lng}=\mathrm{pt}$

19. Taquette SR, Vilhena MM. Uma contribuição ao entendimento da iniciação sexual feminina na adolescência. Psicol Estud Maringá. 2008;13(1):105-14.

20. Centers for Disease Control and Prevention. Youth risk behavior surveillance - United States, 2003. MMWR Morb Mortal Wkly Rep. 2004;53(SS-2):1-96.

21. Centers for Disease Control and Prevention. Young people at risk: HIV/AIDS among America's youth. Atlanta, GA, Centers for Disease Control and Prevention; 2002.

22. OMS 2008 World Health Organization. Inequalities in young people's health. Health Behavior in School- Aged Children. International Report from 2005-2006. Health Police for Children and Adolescents. No 5; 2008.

23. HBSC - Health Behavior in School Aged Children, 2002. Los adolescentes españoles y su salud. Espanha: Ministério de Sanidad y Consumo; 2005.

Recebido em: 05/01/2011

Versão final apresentada em: 03/02/2011

Aprovado em: 04/02/2011 